\title{
DEVELOPMENT OF A GAIT RECOGNITION SYSTEM IN NI LABVIEW PROGRAMMING LANGUAGE
}

\author{
Dániel SALÁNKI, ${ }^{1}$ Kornél SARVAJCZ ${ }^{2}$ \\ University of Debrecen, Faculty of Engineering, Mechatronics Department. Debrecen, Hungary \\ ${ }^{1}$ danisalanki@gmail.com \\ ${ }^{2}$ sarvajcz@eng.unideb.hu
}

\begin{abstract}
Nowadays, the biometric identifier's world is one of the most rapidly developing security technology areas. Within the biometric identification, the research team worked in the area of gait recognition. The research team developed a complex walking recognition system in NI LabVIEW environment that can detect multiple simultaneous reference points using a universal camera and capable of matching a predetermined curve to the collected samples. In the first version, real-time processing was done with a single camera, while in the second, two high-resolution cameras work with post-processing. The program can compare and evaluate the functions that are matched to the reference curve and the current curve in a specific way, whether two walking images are identical. The self-developed gait recognition system was tested on several test subjects by the research team and according to the results, the False Acceptance Rate was zero.
\end{abstract}

Keywords: gait recognition, biometrics, camera system, identifying, analysis.

\section{Introduction}

Nowadays, with world developments, biometric identification systems are becoming more and more widespread. Access control systems, and even the most midrange smartphones have biometric authentication features, and even ID cards can include a person's fingerprint.

There are a number of areas where motion tracking and motion recording devices play an important role. Examples include automation, robotics and computer animation. Different application areas may have different specific requirements, diverse needs may be important. There are places where one or two independent points can be followed, elsewhere, e.g. at character animation, the following points form a complex transformation hierarchy where the complete rigid transformation of each element is required [1].

People often feel that a familiar person is already recognized from a distance by their walking. This common experience is the inspiration for the idea that gait detection systems can be used in security technology. It is easy to create an image of a person's walk, even in public places, without the notice of anything. However, walking is influenced by several factors: footwear, soil, fatigue, current state of mind or any kind of injury [2].

\section{The created gait recognition software [3]}

In 2017, the research team created an initial gait recognition system with one $640 \times 480$ resolution camera in LabVIEW [4]. Now the improved version will be presented below.

The main change of the newer version compared to the previous one is that the program can now handle two cameras instead of one, and instead of real-time processing, the walking is recorded due to the larger system requirements, and after that the gait in the video file is analysed by the program. The cameras were placed side by side to complement each other. A further modification compared to the old version is that instead of point recognition, pattern recognition is performed, which can better filter out the false sensed points, and instead of comparing the coefficients of the functions suited on the gait curves, the definite integral values of the functions are compared. 


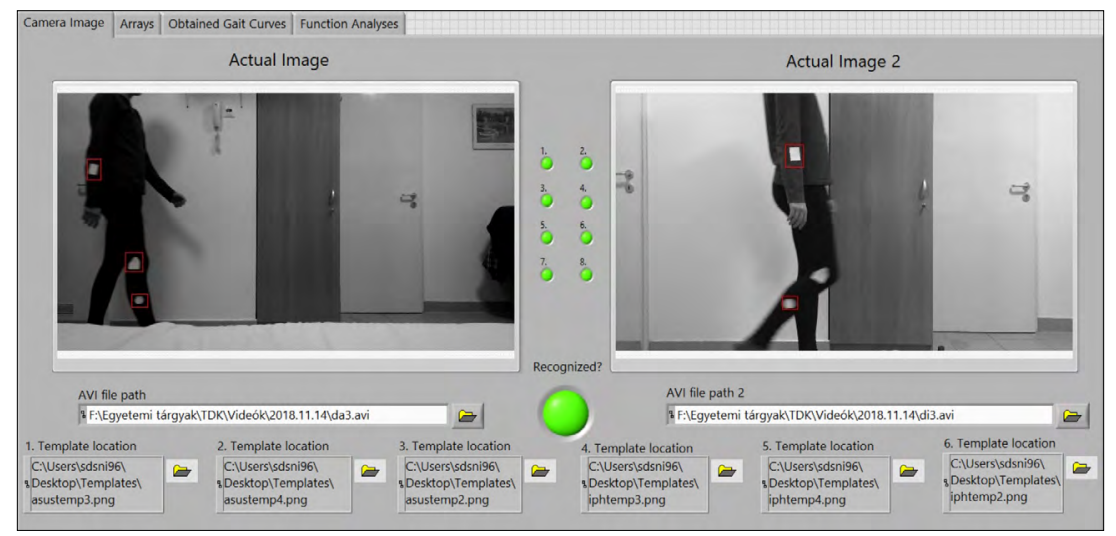

Figure 1. The first tab of the front panel of the gait recognition software

The user interface of the new version of the system is shown in Figure 1. The user can switch between 4 tabs using the Tab Control function. The first page shows the recordings made by the two cameras, which remain in fixed position. Under the two displays the user can choose the computer location of the video files to be selected. Between the two displays exists a large green light that flashes when the test subject is recognized. There are 8 smaller lamps above the larger lamp, which indicate the logical value of 8 comparisons. In the bottom line, the locations of the selectable templates are located, in this case 6, 3 per camera, because in each case 3 templates were placed on the test subject: one on the fore-arm, under the elbow, one on the knee and one on the calf, $36 \mathrm{~cm}$ from the ground. These templates will be searched in the videos. This version no longer requires a Stop button because the program stops automatically after analysing the videos.

On the second tab of the front panel, the coordinates of the detected templates are stored in $\mathrm{X}$ and $\mathrm{Y}$ arrays. On the third tab of the front panel, the resulting gait curves are displayed separately for the first and second video, and the curve drawn by the template on the forearm is evaluated for both videos. During the evaluation, the program fits an equation on the curve drawn by the template on the test subject and calculates its definite integral from 0 to 1920.

The fourth tab of the front panel contains the evaluation of the other walking curves. From left, the first graph shows the function suited on the curve drawn by the first template on the knee shown in the first video, and the second graph describes the function suited on the curve drawn by the template on the calf in the first video, as shown in Figure 2.
The third and fourth graphs suit functions on the curves drawn by the templates on the knee and on the calf respectively in the second video. For the forearm and knee-mounted templates, a second-degree function alignment is performed, because these templates describe a path with a small curvature, so they can be considered straight lines with a good approximation, but for better accuracy, the second-degree coefficient was also considered. However, the curve drawn by the template on the calf cannot be written with a single polynomial, power, or exponential function that the program allows. Based on the observations, about 3 steps with the right foot fit into the camera image, which can be described with 3 second-degree functions. The integration limits are the local maximums of the functions.

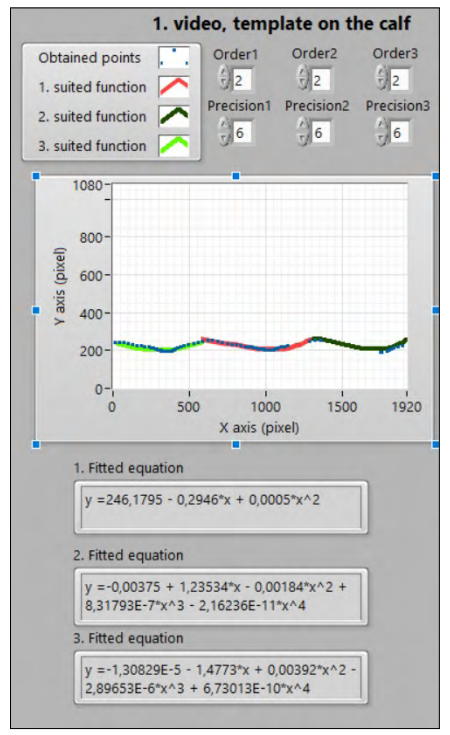

Figure 2. Part of the fourth $t a b$ 
Taking the average of several measurements, the boundaries of the definite integrals of each function were determined. From this, if within $5 \%$ or in some cases $12.5 \%$ differs the current integral value of the function from the predetermined value, then a logical 1 is received from that part of the program. The sum of the definite integral values of the functions suited on the 3 walking curves drawn by the template placed on the calf in the first video was generated, because there was a small standard deviation between the individual values, but the sum of the 3 values was approximately constant during the measurements. In this way, the program makes a total of 8 comparisons, if each gives a logical 1, the green light will flash, and the test subject will be noted as recognized.

\section{Results [3]}

After setting up the camera system, the research group tested it on several subjects. The measurements were made in normal clothing (long-sleeve blouse and trousers). The test subjects took the first step with their left foot. Figure 3. illustrates the results of a test subject known by the program. The first grey column shows the sum of the values of the first 3 blue columns. The blue columns show the results of the first camera video. The values represent the definite integrals of the functions suited on the curves drawn by the templates on the first, second and third functions of the template placed on the calf, the function of the knee, and the curve of the template fitted on the forearm. The green columns show data obtained from the video produced by the second camera in a division similar to the first one. The last column shows the number of the flashing small lights on the front panel.

The program correctly detected the test subject, 14 out of 15 times (Figure 3.). In this case, the false rejection rate (FRR) was $6.25 \%$ (correct detection rate: $93.75 \%$ ).

In the case of a test subject with similar physical parameters to the known test subject, but unknown to the program, the program didn't give him permission even once, so the False Acceptance Rate (FAR) was $0 \%$.

\section{Comparison with other gait recogni- tion systems}

Dacheng Tao, Xuelong Li, Xindong $\mathrm{Wu}$, and Stephen J. Maybank have been testing Gabor-filter based walking detection systems. Gabor filters, named after Dennis Gabor, are linear filters for texture analysis [5]. Gabor filters can be used to amplify linear structures in a given direction. With this method, a detection rate of $92 \%$ was obtained from different views and in different footwear, the detection ratio was $87 \%$ for test subjects with a briefcase [6].

The GEI-based (Gait Energy Image) system, tested by Khalid Bashir, Tao Xiang and Shaogang Gong, was run on multiple databases by the research group without testing cooperative subjects. Based on the CASIASetA database, where there is no package on the test subjects and no big jacket on them, the recognition rate was $100 \%$, however, for CASIASetB and CASIASetC, where the test subjects have a package or are wearing large jackets, the detection rate was $77.8 \%$, and 43.1\% [7].

Chin Poo Lee, Alan W.C. Tan and Shing Chiang Tan were using transient binary patterns (TBP) to

\begin{tabular}{|c|c|c|c|c|c|c|c|c|c|c|c|c|}
\hline & & & & 1. camera & & & & & 2. camera & & & \\
\hline & $1+2+3$ & 1. calf & 2. calf & 3. calf & Knee & Forearm & 1. calf & 2. calf & 3. calf & Knee & Forearm & Lights \\
\hline 1. & 404825 & 155900,9 & 151685,6 & 97238,51 & 689877,1 & 1505048 & 113513,2 & 131300,7 & 57626,29 & 657069,3 & 1582469 & 8 \\
\hline 2. & 404336,2 & 151084,5 & 137148,7 & 116103 & 684607,8 & 1508530 & 112636 & 125737,1 & 58156,21 & 671815,8 & 1588548 & 8 \\
\hline 3. & 407951 & 131239,4 & 135300,6 & 141411 & 678855 & 1497569 & 90849,29 & 121681,8 & 71900,5 & 645783 & 1572135 & 6 \\
\hline 4. & 410753,5 & 148262,4 & 141133,2 & 121357,9 & 684912 & 1492215 & 111130,2 & 121252,9 & 65424,52 & 673045,6 & 1565411 & 8 \\
\hline 5. & 412703,4 & 179398,4 & 136128 & 97177,02 & 684714 & 1500263 & 118396,4 & 124636,5 & 59458,87 & 633641,4 & 1572650 & 8 \\
\hline 6. & 404583,4 & 147187,2 & 143169,9 & 114226,4 & 679971,2 & 1501445 & 107789,5 & 122463,6 & 66480,57 & 685295,5 & 1573187 & 8 \\
\hline 7. & 408340,1 & 134015,8 & 141292,2 & 133032,1 & 680918,3 & 1486649 & 101269,1 & 120697,3 & 67942,65 & 683546,2 & 1555418 & 8 \\
\hline 8. & 406633,9 & 137548,8 & 156790 & 112295,1 & 680750,2 & 1488126 & 110686,4 & 120324,1 & 61604,29 & 647499,3 & 1563726 & 8 \\
\hline 9. & 411615 & 154885,7 & 138469,1 & 118260,3 & 679610,3 & 1486759 & 108561,6 & 127727,8 & 60319,06 & 612740,2 & 1559566 & 8 \\
\hline 10. & 391804,8 & 144159,9 & 138948 & 108696,9 & 676811,2 & 1497082 & 102893 & 124120,1 & 54797,94 & 657944,3 & 1566160 & 8 \\
\hline 11. & 399453,6 & 137030,4 & 158742,8 & 103680,4 & 679787,1 & 1496530 & 107543,7 & 128705,6 & 55152,24 & 604656,2 & 1566528 & 8 \\
\hline 12. & 406380,2 & 144129,1 & 140629,5 & 121621,6 & 674742,4 & 1492289 & 103795,4 & 120809,2 & 66398,06 & 665115,1 & 1573108 & 8 \\
\hline 13. & 402587,5 & 145807,6 & 150664,7 & 106115,2 & 676462,2 & 1484978 & 105895,2 & 128511,1 & 55181,08 & 668137,3 & 1557829 & 8 \\
\hline 14. & 392768,1 & 153320,8 & 136799,3 & 102648 & 675255,9 & 1481227 & 119166,1 & 117615,1 & 60369,81 & 674478 & 1549399 & 8 \\
\hline 15. & 399154,1 & 137491,9 & 141396,9 & 120265,2 & 677788,4 & 1474409 & 100297,6 & 128346,2 & 59830,2 & 624090 & 1546348 & 8 \\
\hline
\end{tabular}

Figure 3. Data from a known test subject's gait 


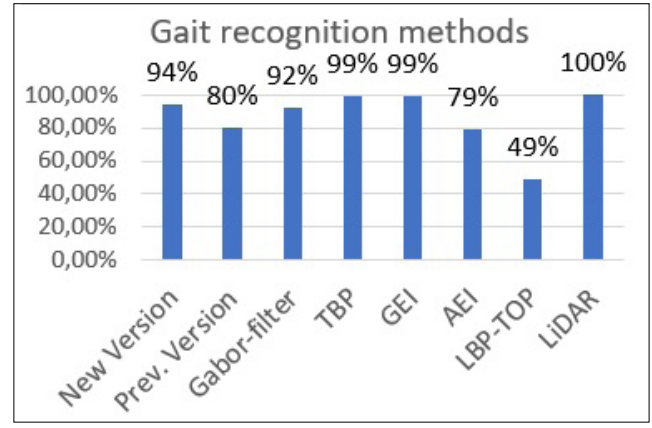

Figure 4. The research group's gait recognition system compared to other systems

develop a gait recognition system and they compared to other methods. The TBP and GEI methods resulted in a 99\% detection rate, the Procrustes Shape Analysis (PSA) with 87\%, 92\% Fourier Descriptive Method, and 79\% Active Energy Image (AEI). The Local Binary Patterns from Three Orthogonal Planes (LBP-TOP), taken from the three orthogonal planes, resulted in only a $49 \%$ detection rate [8].

Bence Gálai and Csaba Benedek studied walking-based personal identification and action recognition with LiDAR sensors (Light Detection and Ranging). Several people have been introduced to their systems at one time, with a $100 \%$ detection rate for up to 5 people, and then gradually reduced to $75 \%$ for 22 people after which it stagnates [9].

Figure 4. summarizes the accuracy of the current and the previous system of the research group and other systems. Based on this, it can be stated that the most accurate systems were the LiDAR (100\%), the TBP (99\%) and the GEI (99\%) based detection systems. The system created by the research team is also good with its $93.75 \%$ recognition rate, while the least efficient is the LBPTOP system with $49 \%$ detection rate.

\section{Conclusions}

In summary, the research group has succeeded in creating a complex walking recognition program in a NI LabVIEW development environment that can detect multiple samples saved as templates using multiple cameras and is able to fit a predefined curve for the collected samples. The program is also able to compare the values of the reference curve and the current curve in a specific way and to evaluate whether the image of the two curves is the same. The self-developed gait recognition system has been tested on a number of test subjects, the program didn't give permission even once to a strange person.

In the future, the processing speed of the gait image could be improved with a camera or software that can be used to instantly save the video in AVI format. The LabVIEW 2014 version used in the research is capable of post-processing only AVI-format videos, so implementing the system in other software (e.g. OpenCV), capable of handling MP4 format, or in which high-quality real-time recording will not reduce the performance, could also improve processing speed.

\section{Acknowledgement}

The work/publication is supported by the EFOP-3.6.116-2016-00022 project. The project is cofinanced by the European Union and the European Social Fund.

\section{References}

[1] Viktor D.: Optikai alapú Motion Capture rendszer. 2011. 5, 7-9.

[2] Boyd J. E., Little J. J.: Biometric Gait Recognition. Springer Verlag Berlin Heidelberg 2005, 19-42.

[3] Salánki D., Sarvajcz K., Husi G.: Járásfelismerés fejlesztése NI LabVIEW környezetben. Debreceni Egyetem Müszaki Kar, Mechatronikai mérnök BSc, szakdolgozat, 2018.

[4] Salánki D: Járásfelismerés fejlesztése NI LabVIEW környezetben. Debreceni Egyetem Müszaki Kar, Kari TDK, Debrecen, 2017.

[5] Fogel I., Sagi D.: Gabor filters as texture discriminator. Biological Cybernetics, 1989, 61.

[6] Dacheng Tao, Xuelong Li, Xindong Wu, Maybank S. J.: General Tensor Discriminant Analysis and Gabor Features for Gait Recognition. IEEE transactions on pattern analysis and machine intelligence, 2007. 1-35.

[7] Khalid Bashir, Tao Xiang, Shaogang Gong: Gait recognition without subject cooperation. Pattern Recognition Letters, 2010. 2052-2060.

[8] Chin Poo Lee, Alan W.C. Tan, Shing Chiang Tan: Gait recognition with Transient Binary Patterns. J. Vis. Commun. Image R., 2015. 69-77.

[9] Gálai B., Benedek Cs.: Járás alapú személyazonositás és cselekvésfelismerés LiDAR szenzorokkal, 2017. 